\title{
Sobrecarga GerAdA PElo CONVÍVIO COM O PORTADOR DE ESQUIZOFRENIA: A ENFERMAGEM CONSTRUINDO O CUIDADO À FAMÍLIA
}

\author{
Mariana Silva Gomes ${ }^{1}$; \\ Rosâne Mello
}

\begin{abstract}
A esquizofrenia é doença mental crônico-degenerativa, em que o portador apresenta dificuldades para o autocuidado. Em razão da desinstitucionalização, a vivência cotidiana acarreta sobrecarga aos familiares. O objetivo, aqui, foi analisar o grau de sobrecarga do principal cuidador que convive com o portador de esquizofrenia, em um hospital de emergência psiquiátrica do Estado do Rio de Janeiro. Trata-se de pesquisa descritiva, com abordagem quantitativa. Participaram dez familiares, em um período de quinze dias para coleta de dados. Utilizou-se a escala de sobrecarga dos familiares de pacientes psiquiátricos, analisados pela escala Likert. Foram encontradas sobrecargas elevadas na rotina diária do familiar e intenso abalo da sua saúde mental, sendo fundamental que a enfermagem inclua as famílias no tratamento, diminuindo as sobrecargas.
\end{abstract}

Descritores: Esquizofrenia; Enfermagem Psiquiátrica; Saúde da Família.

\footnotetext{
${ }^{1}$ Aluna do Curso de Graduação em Enfermagem, Escola de Enfermagem Alfredo Pinto, Universidade Federal do Estado do Rio de Janeiro, RJ, Brasil. E-mail: mariana-sgomes@hotmail.com.

${ }^{2}$ Enfermeira, Doutor em Enfermagem, Professor Adjunto, Escola de Enfermagem Alfredo Pinto, Universidade Federal do Estado do Rio de Janeiro, RJ, Brasil. E-mail: rosane.dv@gmail.com.
} 


\section{OVERLOAD GENERATED BY THE INTERACTION WITH SCHIZOPHRENIA PATIENTS: NURSING BUILDING FAMILY CARE}

Schizophrenia is a chronic degenerative mental disease, in which the patient has difficulty in self care. Due to deinstitutionalization, the daily life brings burden to family. The goal here was to analyze the degree of overload of the primary caregiver living with the holder of schizophrenia in a psychiatric emergency hospital in the State of Rio de Janeiro. This is a descriptive research with quantitative approach. The participants were ten families in a period of fifteen days for data collection. We used the scale of burden of psychiatric patients' relatives, analyzed by the Likert scale. Overloads were found high in the familiar daily routine and intense shock of their mental health, being fundamental that nursing include families in treatment, reducing the overload.

Descriptors: Schizophrenia; Psychiatric Nursing; Family Health.

\section{RECARGO GENERADO POR EL CONVIVIO CON EL PORTADOR DE ESQUIZOFRENIA: LA ENFERMERÍA CONSTRUYENDO EL CUIDADO A LA FAMILIA}

La esquizofrenia es enfermedad mental crónico-degenerativa, en que el portador presenta dificultades para el autocuidado. En razón de la desinstitucionalización, la vivencia cotidiana acarrea recargo a los familiares. El objetivo, aquí, fue analizar el grado de recargo del principal cuidador que convive con el portador de esquizofrenia, en un hospital de emergencia psiquiátrica de la Provincia de Rio de Janeiro. Se trata de pesquisa descriptiva, con abordaje cuantitativo. Participaron diez familiares, en un período de quince días para recogida de datos. Se utilizó la escala de recargo de los familiares de pacientes psiquiátricos, analizados por la escala Likert. Fueron encontrados recargos elevados en la rutina diaria del familiar e intenso estremezco de su salud mental, siendo fundamental que la enfermería incluya las familias en el tratamiento, apocando los recargos.

Descriptores: Esquizofrenia; Enfermería Psiquiátrica; Salud de la Família.

\section{Introdução}

A esquizofrenia é doença mental crônicodegenerativa, na qual as pessoas adoecidas têm dificuldade para reconhecer a realidade, de se comportar dentro dos parâmetros sociais de normalidade e até mesmo de realizar tarefas de autocuidado e higiene pessoal. É a mais grave das doenças mentais, acomete em torno de dois milhões de brasileiros e é cercada por tabus e preconceitos, em razão do desconhecimento da maioria da população acerca dessa doença $a^{(1)}$.

Considerando a família como uma unidade provedora de cuidados das situações de saúde e doença dos seus membros, o lugar do reconhecimento das diferenças e a sede das primeiras trocas afetivas, essa se encontra em contato constante e diário com o portador de esquizofrenia ${ }^{(2)}$.

Dessa forma, a família sofre intensamente com a situação da pessoa adoecida, vivenciando sentimentos de aflição, depressão, isolamento, tristeza crônica, culpa e angústia. A presença do transtorno provoca ruptura da rotina existencial da família, na qual o principal cuidador passa a colocar suas próprias necessidades e vontades em segundo plano, tornando-se sobrecarregado por arcar com os ônus gerados pela doença $a^{(3)}$.

Por outro lado, há famílias que cultivam sentimentos de negação, revolta e atitude crítica em relação ao portador 
de sofrimento psíquico. Porém, a vivência cotidiana faz com que esses familiares também sofram com os desgastes provocados pelo transtorno, pois o impacto emocional que o transtorno psíquico traz aos familiares é, muitas vezes, tão intenso quanto aquele que atinge o paciente ${ }^{(1)}$.

O termo "sobrecarga familiar" fundamenta-se no impacto provocado pela presença do portador de sofrimento mental junto ao ambiente familiar, podendo envolver aspectos econômicos, práticos e emocionais a que se encontram submetidos aqueles familiares encarregados do cuidado necessário e exigido pelo paciente ${ }^{(4)}$.

A sobrecarga sentida pela família caracteriza-se por dimensões objetivas e subjetivas. A sobrecarga objetiva se refere às consequências negativas, concretas e observáveis, resultante da presença do doente mental na família. Quando se discute a sobrecarga objetiva, consideram-se as tarefas cotidianas extras que eles precisam realizar para suprir as necessidades do doente mental, as perdas financeiras, as mudanças radicais na rotina social, familiar e profissional do familiar, bem como o suporte que o esquizofrênico recebe de seus familiares ${ }^{(5-6)}$.

Em contrapartida, o aspecto subjetivo da sobrecarga é definido pela percepção do familiar acerca da situação, sua reação emocional, seu sentimento em estar sofrendo uma sobrecarga, bem como o abalo da sua saúde mental ${ }^{(5)}$.

Diante do exposto, considera-se relevante estudar a sobrecarga familiar resultante da convivência com o portador de esquizofrenia, visto que a atual política de saúde mental brasileira baseia-se no retorno e na permanência da pessoa com transtorno psíquico em seu núcleo familiar e social, englobando o lazer, a comunidade e o trabalho ${ }^{(7)}$.

Em virtude disso, faz-se necessário que os profissionais de enfermagem incluam a família no cuidado à pessoa com transtorno mental, conheça e valorize as possíveis sobrecargas existentes na família e estabelça estratégias para diminuí-las.

Delineou-se, aqui, o objetivo do estudo: analisar o grau de sobrecarga do principal cuidador que convive com o portador de esquizofrenia, em um hospital de emergência psiquiátrica no Estado do Rio de Janeiro.

\section{Material e Métodos}

Optou-se por pesquisa descritiva, com abordagem quantitativa.

O cenário para a coleta de dados foi um hospital municipal de emergência psiquiátrica, localizado no Estado do Rio de Janeiro. A pesquisa foi realizada nas reuniões de famílias, onde os pacientes não participavam, apenas o profissional de saúde e os familiares, e o período da coleta de dados foi de quinze dias.

Os sujeitos foram dez familiares - principais cuidadores - que conviviam com a pessoa com diagnóstico médico de esquizofrenia. Dentre eles, incluíram-se familiares de ambos os sexos, a idade variou entre 27 e 78 anos, sendo predominante a faixa etária de 41 a 50 anos. A totalidade dos participantes justifica-se pelos frequentadores assíduos das reuniões de famílias.
Os dados foram obtidos através de dois instrumentos: o instrumento de contextualização do familiar e a escala de sobrecarga dos familiares de pacientes psiquiátricos.

O primeiro instrumento constitui-se de uma entrevista estruturada, contemplando questões como: dados de identificação do familiar, a relação do familiar com o portador do sofrimento psíquico e a relação do familiar com o serviço.

Já o segundo instrumento trata-se de escala adaptada e validada para o Brasil, com a finalidade de avaliar a sobrecarga do principal cuidador do paciente psiquiátrico ${ }^{(8)}$.

Essa escala possui 41 itens quantitativos, sendo avaliadas diversas dimensões da vida do familiar, organizadas em subescalas. São elas: A) assistência na vida cotidiana, B) supervisão aos comportamentos problemáticos, C) gastos financeiros, D) impacto na rotina diária e E) preocupação com o paciente.

Para o escalonamento das respostas referentes às sobrecargas, foi adotada a escala Likert. Sendo que as questões referentes à sobrecarga objetiva e à avaliação das preocupações do familiar com o paciente as alternativas de respostas contêm cinco pontos. Desses, 1=nenhuma vez, $2=$ menos que uma vez por semana, $3=$ uma ou duas vezes por semana, $4=$ três a seis vezes por semana e $5=$ todos os dias.

Para as questões referentes à sobrecarga subjetiva, em relação ao grau de incômodo, as opções de respostas contêm quatro pontos, sendo que $1=$ nem um pouco, $2=$ muito pouco, $3=$ um pouco e $4=$ muito.

O estudo foi autorizado pela unidade, como também, submetido e aprovado pelo Comitê de Ética em Pesquisa do próprio hospital.

Anteriormente ao início da entrevista, todos os familiares manifestaram sua aceitação em participar do estudo, por escrito, mediante assinatura do termo de consentimento livre e esclarecido ${ }^{(9)}$.

Para a análise dos dados do instrumento de contextualização do familiar, realizou-se a análise descritiva dos resultados. Já para a verificação da sobrecarga dos familiares, da escala de sobrecarga dos familiares de pacientes psiquiátricos, analisou-se a porcentagem de respostas para cada item das subescalas, considerando que: nas questões referentes à sobrecarga objetiva e na avaliação das preocupações do familiar com o paciente, as respostas acima de 4 indicam sobrecarga elevada. Já nas questões referentes à sobrecarga subjetiva, as respostas acima de 3 evidenciam sobrecarga elevada dos principais cuidadores ${ }^{(10)}$.

A subescala $C$ não se aplica aos cálculos de porcentagem, pois se refere a valores em dinheiro das despesas com o paciente, e avalia as questões econômicas do grupo familiar ${ }^{(8)}$.

\section{Resultados e Discussão}

\section{Caracterização dos sujeitos}

A totalidade de familiares morando com o paciente e sendo os principais cuidadores contribui para aumentar 
a frequência de tarefas de assistência prestadas ao doente, visto que é sobre os principais cuidadores que recaem as tarefas mais árduas do cuidado ao esquizofrênico, que se encontra debilitado para realizar seus cuidados pessoais ${ }^{(10)}$.

Em relação ao grau de parentesco do familiar com o portador de esquizofrenia, as mães foram identificadas como as principais cuidadoras dos pacientes $(60 \%)$, ocorrendo o predomínio do sexo feminino entre os sujeitos.

A tarefa de cuidar do doente mental vem sendo atribuída às mulheres e, ao pesquisar a voz das mulheres que cuidam de doentes mentais, constatou-se que elas estão sujeitas a diversos reflexos, decorrentes do seu papel, e expressam dificuldades para lidar com a pessoa adoecida na família, principalmente em crise, quando os esquizofrênicos expressam delírios e alucinações, gerando intensos conflitos na família ${ }^{(11-12)}$.

O grau de escolaridade predominante dos familiares foi o $1^{\circ}$ grau incompleto $(80 \%)$, com renda mensal média de $\mathrm{R} \$ 495,00$. Todos os entrevistados sustentavam a casa.
Algumas características dos cuidadores apontam aumento do desgaste familiar, dentre elas, a baixa escolaridade e o baixo nível socioeconômico ${ }^{(13)}$.

Além disso, todos os sujeitos participantes faziam atendimentos no serviço, do tipo reuniões de famílias. E a maioria deles (70\%) submetia-se a tratamento medicamentoso, com antidepressivos.

Alguns estudos corroboram esse resultado da pesquisa, ao ressaltar que as famílias sofrem intenso desgaste com a doença mental do ente querido, vivenciando sentimentos de aflição e tristeza, manifestando-os através de quadros psicopatológicos por estresse, onde a depressão é o mais comum dentre eles ${ }^{(14)}$.

\section{Sobrecarga objetiva}

Os resultados relacionados à sobrecarga objetiva estão dispostos na Tabela 1 .

Tabela 1 - Porcentagens de respostas dos familiares para cada item das questões referentes à sobrecarga objetiva

\begin{tabular}{|c|c|c|c|}
\hline \multirow{2}{*}{ Subescalas } & \multirow{2}{*}{ Itens } & \multirow{2}{*}{$\begin{array}{c}\text { Respostas } 1,2 \text { e } 3 \\
(\%)\end{array}$} & \multirow{2}{*}{$\begin{array}{c}\text { Respostas } 4 \text { e } 5 \\
(\%)\end{array}$} \\
\hline & & & \\
\hline \multirow[t]{9}{*}{ A- Assistência na vida cotidiana } & Higiene & - & 100 \\
\hline & Medicação & - & 100 \\
\hline & Tarefas de casa & 60 & 40 \\
\hline & Compras & 20 & 80 \\
\hline & Alimentação & 30 & 70 \\
\hline & Transporte & 30 & 70 \\
\hline & Dinheiro & 40 & 60 \\
\hline & Ocupação do tempo & 60 & 40 \\
\hline & Consultas médicas & 30 & 70 \\
\hline \multirow[t]{8}{*}{ B- Supervisão aos comportamentos problemáticos } & Comportamentos problemáticos & 40 & 60 \\
\hline & Demanda excessiva de atenção & 30 & 70 \\
\hline & Perturbação noturna & 40 & 60 \\
\hline & Heteroagressão & 60 & 40 \\
\hline & Autoagressão & 80 & 20 \\
\hline & Bebidas alcoólicas & 90 & 10 \\
\hline & Excesso de cigarros, alimentos e líquidos & 90 & 10 \\
\hline & Abuso de drogas & 90 & 10 \\
\hline \multirow[t]{4}{*}{ D- Impacto na rotina diária do cuidador } & Atrasos ou ausências a compromissos & 40 & 60 \\
\hline & Alteração do lazer & 40 & 60 \\
\hline & Alteração do serviço/rotina de casa & 30 & 70 \\
\hline & Redução da atenção aos outros familiares & 30 & 70 \\
\hline
\end{tabular}

Obs: 1=nenhuma vez; 2=menos que uma vez por semana; $3=$ uma ou duas vezes por semana; $4=$ rês a seis vezes por semana; $5=$ todos os dias.

De acordo com a subescala A, a maioria dos cuidadores respondeu que prestam assistência na vida cotidiana do portador do sofrimento psíquico com elevada frequência, evidenciando elevada sobrecarga objetiva ao familiar.

Nesse sentido, a rigorosa administração de medicamentos, as dificuldades do esquizofrênico nas tarefas de higiene e as suas limitações em gerenciar sua própria vida são situações desgastantes ao cuidador, fazendo com que esse tenha que supervisionar e ajudar o paciente com intensa frequência nessas atividades, causando aumento da sobrecarga ao familiar ${ }^{(15)}$.
Analisando a subescala B, observou-se que a maioria dos familiares não realizava supervisões aos comportamentos problemáticos com intensa frequência, prevalecendo sobrecarga baixa nessa subescala.

Uma explicação para esse resultado seria que, ao longo das reuniões de famílias, foi possível constatar que a imensa maioria dos esquizofrênicos não apresentava comportamentos agressivos para com os outros, nem para consigo mesmo, assim como não abusavam de bebidas alcoólicas e drogas.

Ao analisar a subescala $D$, foi constatado que a 
maioria dos familiares sofre o impacto nas rotinas diárias, visto responderam que são prejudicados em suas rotinas, com elevada frequência, acarretando-lhes alta sobrecarga.

Ratificando esse resultado, a família que convive com esse transtorno psíquico, apresenta sobrecarga em suas rotinas diárias, no sentido de que precisam assumir as atividades domésticas do doente, que estão prejudicadas em razão da complexa sintomatologia da doença, a qual engloba a desorientação das atividades do dia a dia, fazendo com que o familiar ausente-se do trabalho, dos seus compromissos sociais e direcione sua atenção para o cuidado ao doente mental ${ }^{(16)}$.
Além disso, a alteração do lazer do familiar é explicado pelo grande estigma e preconceitos que envolvem a doença, na qual o esquizofrênico é excluído pela sociedade e, consequentemente, seu principal cuidador também sofre esse preconceito, ocasionando afastamento das atividades de lazer ${ }^{(16)}$.

\section{Sobrecarga subjetiva}

Os resultados relacionados à sobrecarga subjetiva estão dispostos na Tabela 2.

Tabela 2 - Porcentagem de respostas dos familiares para cada item das questões referentes à sobrecarga subjetiva

\begin{tabular}{|c|c|c|c|}
\hline \multirow{2}{*}{ Subescalas } & \multirow{2}{*}{ Itens } & \multirow{2}{*}{$\begin{array}{c}\text { Respostas } 1 \text { e } 2 \\
(\%)\end{array}$} & \multirow{2}{*}{$\begin{array}{c}\text { Respostas } 3 \text { e } 4 \\
(\%)\end{array}$} \\
\hline & & & \\
\hline \multirow[t]{9}{*}{ A- Assistência na vida cotidiana } & Higiene & 10 & 90 \\
\hline & Medicação & - & 100 \\
\hline & Tarefas de casa & 70 & 30 \\
\hline & Compras & 20 & 80 \\
\hline & Alimentação & 60 & 40 \\
\hline & Transporte & 30 & 70 \\
\hline & Dinheiro & 40 & 60 \\
\hline & Ocupação do tempo & 60 & 40 \\
\hline & Consultas médicas & 30 & 70 \\
\hline \multirow{8}{*}{$\begin{array}{l}\text { B- Supervisão aos } \\
\text { comportamentos problemáticos }\end{array}$} & Comportamentos problemáticos & 40 & 60 \\
\hline & Demanda excessiva de atenção & 30 & 70 \\
\hline & Perturbacão noturna & 40 & 60 \\
\hline & Heteroagressão & 60 & 40 \\
\hline & Autoagressão & 80 & 20 \\
\hline & Bebidas alcoólicas & 90 & 10 \\
\hline & Excesso de cigarros, alimentos e líquidos & 90 & 10 \\
\hline & Abuso de drogas & 90 & 10 \\
\hline
\end{tabular}

Obs: $1=$ nem um pouco; $2=$ muito pouco; $3=$ um pouco; $4=$ muito.

A subescala A demonstrou que a maioria dos familiares sentiu-se extremamente incomodada ao prestar assistência na vida cotidiana do paciente, indicando-lhes elevada sobrecarga subjetiva, ou seja, abalo da sua saúde mental.

Dessa forma, a família sente bastante o impacto em ter que suprir as necessidades do doente mental, abdicando da sua própria vida e dos seus desejos para cuidar do portador de esquizofrenia, que demanda intensa atenção pela sua condição de limitação em administrar seus bens e sua vida pessoal ${ }^{(16)}$.

Ao analisar a subescala $B$, nas questões que tratam de agressão, tabagismo, alcoolismo e drogas, foi observado que a reduzida presença desses comportamentos, nos esquizofrênicos do estudo, acarreta sobrecarga baixa ao familiar, ou seja, diminuição do seu impacto, quando considerados em conjunto ${ }^{(10)}$.

Os resultados da subescala E estão distribuídos na Tabela 3.

Tabela 3 - Porcentagem de respostas dos familiares para cada item das questões da subescala E

\begin{tabular}{|c|c|c|c|}
\hline \multirow{2}{*}{ Subescala } & \multirow{2}{*}{ Itens } & \multirow{2}{*}{$\frac{\text { Respostas } 1,2 \text { e } 3}{(\%)}$} & \multirow{2}{*}{$\begin{array}{c}\text { Respostas } 4 \text { e } 5 \\
(\%)\end{array}$} \\
\hline & & & \\
\hline \multirow[t]{7}{*}{ E- Preocupações com o paciente } & Segurança física & - & 100 \\
\hline & Qualidade do tratamento & 20 & 80 \\
\hline & Vida social & 30 & 70 \\
\hline & Saúde & 10 & 90 \\
\hline & Moradia & 90 & 10 \\
\hline & Finanças & 40 & 60 \\
\hline & Futuro & 10 & 90 \\
\hline
\end{tabular}

Obs: $1=$ nunca; $2=$ raramente; $3=$ às vezes; $4=$ frequentemente; $5=$ sempre ou quase sempre. 
De acordo com a análise da subescala E, verificouse que a imensa maioria dos familiares preocupava-se intensamente com o paciente, acarretando-lhes elevada sobrecarga.

A família do portador de esquizofrenia sentese insegura em relação à qualidade do tratamento que está sendo prestado ao paciente, medo em relação à segurança física do paciente, pois, nos momentos de crise, há exacerbação dos movimentos corporais, intensa preocupação com a saúde e com o futuro do familiar adoecido, principalmente como seria sua situação financeira, se não fosse o cuidador ${ }^{(1)}$.

Em relação aos resultados da subescala $C$, a média de gastos mensais do familiar com o paciente foi de $\mathrm{R} \$ 325,00$. Considerando a renda mensal média das famílias ( $\mathrm{R} \$ 495,00)$, e que a maioria dos pacientes não contribuía com as despesas mensais, foi possível detectar a presença de sobrecarga financeira nos cuidadores. Dessa forma, a família que convive com o esquizofrênico, costuma sentir sobrecarga financeira, considerando a incapacidade produtiva como um dos sintomas da doença, ou seja, a pessoa adoecida deixa de trabalhar, ocorrendo aumento dos gastos para o principal cuidador, resultante da compra de medicamentos, alimentação e outros gastos que geram alteração no orçamento familiar ${ }^{(16)}$.

\section{A enfermagem no cuidado às famílias}

Com o processo de desinstitucionalização, a família foi incluída na assistência ao portador de transtorno mental, visando melhor recuperação do doente, e a devida participação da sociedade e da própria família no tratamento, redirecionando o modelo assistencial em saúde mental ${ }^{(17)}$.

As diminuições das sobrecargas sentidas pelo familiar são consideradas significativas quando o principal cuidador participa de programas de suporte familiar, expondo suas angústias, dúvidas sobre a doença e trocando informações relevantes sobre as situações difíceis, geradas pela convivência com o portador de esquizofrenia ${ }^{(13)}$.

Diante desse contexto, é fundamental que os profissionais de enfermagem desenvolvam ações de acolhimento aos familiares, através de atendimento individual, atendimento nuclear de famílias, grupos psicoeducacionais, de forma que possam promover a escuta, esclarecimento acerca da doença, auxílio na gestão da vida cotidiana e estabelecimento de estratégias de enfrentamento do próprio sofrimento psíquico do familiar e do doente ${ }^{(18)}$. Sendo assim, o empoderamento do familiar nos momentos de crise, a queda da sobrecarga e a convivência familiar harmônica constituem consequências de apoio adequado às famílias.

Além disso, é necessário estimular à adesão do familiar ao tratamento, visto que uma família cooperativa reduz o risco de recaída e, consequentemente, as instabilidades no âmbito familiar ${ }^{(1)}$.

Reforçando essas observações, as reuniões de famílias, chamadas "grupos psicoeducacionais" para familiares, têm contribuído para a convivência menos conflituosa entre o cuidador e o portador do sofrimento psíquico, uma vez que auxilia as famílias quanto ao entendimento da doença, gerando mais segurança e otimismo ao familiar ${ }^{(19)}$.

\section{Conclusões}

Considerando o núcleo familiar o principal meio de convívio do portador de esquizofrenia, a família assume maiores responsabilidades, o que inegavelmente acarreta alterações nas atividades cotidianas, no orçamento familiar e maiores preocupações, gerando sobrecargas ao principal cuidador.

Diante disso, certifica-se que a família precisa se sentir preparada para receber e cuidar do doente mental no ambiente familiar, orientada pelos profissionais de saúde mental, de forma que possa expor livremente todos os seus problemas e segura para enfrentar situações complicadas, advindas da convivência com o portador do sofrimento psíquico.

Ratifica-se, portanto, que as intervenções psicossociais de cuidado aos familiares de indivíduos com esquizofrenia precisam ser cada vez mais adotadas nos serviços de saúde mental. Para que essas intervenções sejam eficazes para os familiares, faz-se necessário obter participação colaborativa da família e reconhecer as dificuldades pelas quais ela passa a ter com a presença de um membro com grave incapacitação mental.

A partir do que foi discutido, acredita-se que o profissional de saúde mental, ao trabalhar em conjunto com as famílias, com os pacientes e os serviços, precisa saber detectar o grau de sobrecarga dos principais cuidadores da pessoa adoecida, desenvolver propostas de cuidado às famílias, minimizando os encargos familiares e incrementando a qualidade de vida do portador de esquizofrenia, assim como do grupo familiar.

\section{Referencias}

1. Palmeira L, Geraldes MT, Bezerra AB. Entendendo a Esquizofrenia: como a família pode ajudar no tratamento? Rio de Janeiro: Interciência; 2009.

2. Castilho T. Família e relacionamento de gerações. In: Congresso Internacional Co-Educação de Gerações. 2003; São Paulo: SESC. [acesso 03 fev 2010]. Disponível em: http://www.sescsp.org.br/sesc/images/upload/ conferencias/94.rtf

3. Zanetti AC, Galera S. O impacto da esquizofrenia para a família. Rev Gaúch Enferm. 2007;28(3):385-92.

4. Pegoraro RF, Caldana, RH. Sobrecarga de familiares de usuários de um Centro de Atenção Psicossocial. Psicol Estudo. 2006;11(3):569-77.

5. Maurin JT, Boyd CB. Burden of mental illness on the family: a critical review.

Arch Psychiatr Nurs. 1990;4(2):99-107.

6. Tessler RC, Gamache GM. Family experiences with mental illness. Auburn House: Westport; 2000.

7. Mello R. A construção do cuidado à família e a consolidação da reforma psiquiátrica. Rev Enferm UERJ. 2005;13(3):390-6.

8. Bandeira M, Calzavara MG, Varella AA. Escala 
de sobrecarga dos familiares de pacientes psiquiátricos: adaptação transcultural para o Brasil (FBIS-BR). J Bras Psiquiatr. [periódico na Internet]. 2005. [acesso 04 nov 2009]; 54(3):206-14. Disponível em: http://www.ufsj.edu. br/lapsam/sobrecarga_familiar.php

9. Hardy E, Bento SF, Osis MJ. Consentimento Informado Normatizado pela Resolução 196/96: Conhecimento e Opinião de Pesquisadores Brasileiros. RBGO. 2002;24(1):59-65.

10. Barroso SM, Bandeira $M$, Nascimento E. Sobrecarga de familiares de pacientes psiquiátricos atendidos na rede pública. Rev Psiquiatr Clín. 2007;34(6):270-7.

11. Ornellas CP. O paciente excluído: história e críticas das práticas médicas de confinamento. Rio de Janeiro: Universidade Federal de Santa Catarina; 1997. p. 150-231. [acesso 17 mar 2010]. Disponível em: http:// www.cfh.ufsc.br/ labhiss/exclud2.html

12. Gonçalves AM, Sena RR. A reforma psiquiátrica no Brasil: Contextualização e reflexos sobre o cuidado com o doente mental na família. Rev. Latino-Am. Enfermagem. 2001;9:48-55.

13. Loukissa D. Family burden in chronic mental illness: a review of research studies. J Adv Nurs. 1995;21(2):248-55.

14. Scherer ZP, Scherer EA. O doente mental crônico internado: uma revisão da literatura. Rev. Latino-Am. Enfermagem. 2001;9(4):56-61.

15. Spaniol L, Zipple AM, Lockwood D. The role of the family in psychiatric rehabilitation. Schizophrenia Bull Rockville. 1992;18:341-8.

16. Koga M. Convivência com a pessoa portadora de esquizofrenia: Sobrecarga familiar [dissertação de mestrado]. Ribeirão Preto (SP): Escola de Enfermagem de Ribeirão Preto da Universidade de São Paulo; 1997.

17. Ministério da Saúde (BR). Lei No 10.216 , de 6 de abril de 2001. Dispõe sobre a proteção e os direitos das pessoas portadoras de transtornos mentais e redireciona o modelo assistencial em saúde mental. Brasília (DF): Ministério da Saúde; 2001.

18. Scazufca M. Abordagem familiar em esquizofrenia. Rev Bras Psiquiatr. 2000;22, Suppl 1:50-2.

19. Yacubian J. Grupos psicoeducacionais para familiares. São Paulo: Associação Brasileira de familiares, amigos e portadores de transtornos afetivos; 2009 [acesso 01 de jun 2010]. Disponível em: http://www.abrata.org.br/ site/artigos/artigos.asp?vLink=artigos\&artigoId $=12$

\section{Como citar este artigo:}

Gomes MS, Mello R. Sobrecarga gerada pelo convívio com o portador de esquizofrenia: a enfermagem construindo o cuidado à família. SMAD, Rev. Eletrônica Saúde Mental Álcool Drog. (Ed. port.). jan.-abr. 2012 [acesso: Disponível em:

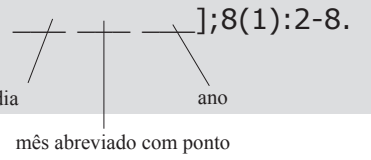

